tension baisse d'une façon continue pendant 2 ou 3 heures environ. $\mathrm{Au}$ bout de ce temps, la tension superficielle ne varie plus ; elle a atteint une valeur stable (valeur statique).

$3^{\circ}$ Entre $20^{\circ}$ et $0^{\circ}$, le refroidissement influe -sur la tension superficielle. Aux environs de $20^{\circ}$, la valeur de la tension superficielle reste sensiblement identique, mais si on abaisse la température respectivement à $10^{\circ}$, puis à $0^{\circ}$, on constate une forte dépression. A $0^{\circ}$, la tension superficielle a atteint sa valeur minima.

\title{
A PROPOS DE LA DÉSACIDIFICATION ÉLECTRIQUE DU LAIT
}

\author{
JEAN PIEN \\ Ingénieur-Chimiste (I. C. R.) \\ Docteur ès-Sciences \\ Directeur des laboratoires \\ des "Fermiers Réunis".
}

par

Depuis quelques années déjà on parle de la réduction électrique de l'acidité du lait. Depuis quelques mois même, on peut voir en service et expérimenter les appareils réalisant cette opération sur des bases industrielles.

Etant donné l'intérêt évident que présenterait en fromagerie et dans la préparation de certains sous-produits du lait, la solution de ce problème de la désacidification par des voies autres que la neutralisation chimique, nous avons tenu à expérimenter les appareils offerts actuellement dans ce but à l'industrie laitière.

Cet article qui relatera les résultats de nos essais aura pour objet :

$1^{\circ}$ De décrire succinctement l'opération de la désacidification électrique ;

$2^{\circ}$ De montrer quelles modifications surviennent dans la composition du lait au cours de cette opération ;

$3^{\circ}$ D'en déduire une théorie provisoire de la désacidification électrique ;

$4^{\circ}$ De montrer les avantages et les inconvénients du procédé.

$$
* * *
$$

\section{ALLURE GÉNERALE DU PHÉNOMẼNE}

Les appareils destinés à réaliser industriellement la désacidification électrique du lait ont été décrits dans cette revue $[1,2]$. Nous croyons done inutile d'y revenir.

Qu'il nous suffise de rappeler que ces appareils sont constitués par de véritables cuves à électrolyse consistant en un bac en grès 
où plongent des séries d'électrodes en aluminium. Le lait passe entre ces électrodes et subit l'action d'une différence de potentiel.

Le voltage et la densité du courant sont réglables ainsi que le débit du lait suivant les effets à obtenir.

Deux faits importants s'imposent à l'observation lors d'une opération de désacidification électrique :

$1^{0}$ Le lait mousse abondamment. Cette mousse était facile à prévoir puisque le passage du courant détermine la décomposition de l'eau du lait. Mais elle est extrêmement abondante. Notons toutefois que l'appareil est établi de telle manière que la mousse est assez. rapidement résorbée.

$2^{\circ}$ Le deuxième effet visible du passage du courant est une préeipitation notable de la caséine qui, sous forme de caillebotte, se dépose contre les électrodes ou au fond de la cuve ou bien est entraînée par les mousses.

\section{MODIFICATIONS SURVENUES DANS LE LAIT AU COURS DU TRAITEMENT}

\section{Acidité.}

On note effectivement-une chute d'acidité d'autant plus importante que le débit du lait était plus faible ou que la densité du courant était plus élevée.

On a prétendu que l'acide lactique était décomposé au cours de l'opération. Il n'en est rien. L'acide lactique n'est pas détruit par l'électrolyse dans les conditions où elle se produit ici.

On le met facilement en évidence en dosant l'acide lactique non par acidimétrie, mais par iodométrie après l'oxydation classique en éthanal par le permanganate en milieu sulfurique. Ce dosage effectué avant et après le traitement fournit les mêmes chiffres. Et ce résultat a été obtenu même dans le cas de laits titrant $40^{\circ}$ Dornic avant le traitement et entièrement neutralisés électriquement.

A quoi est due la diminution de l'acidité ?

a) D'une part (mais en partie seulement) à la précipitation anodique de la caséine. Ce protide, de réaction amphotérique dans le lait, ne figure plus comme acidité titrable dans le lait après son insolubilisation. Cette "disparition " entraîne une diminution de l'acidité titrable.

Toutefois, en dosant la caséine ainsi précipitée on acquiert aisément la certitude que ce mécanisme ne suffit pas à expliquer le phénomène. Il faut faire intervenir une autre cause de désacidification.

b) Cette autre cause est, à notre avis, la fixation de l'acide lactique libre, présent dans les laits acides, sur les bases mises en 
liberté au cours de réactions secondaires qui seront exposées ciaprès.

Mais cette saturation de l'acide lactique n'est pas une décomposition et les radicaux lactiques fixés se retrouvent intégralement après l'opération.

\section{Caséine.}

La désacidification électrique du lait entraîne une perte sensible de caséine qui se préeipite sur les plaques anodiques d'une façon importante surtout dans le cas de grandes densités de courant.

Prenons un exemple : dans le cas d'un traitement très ménagé (lait à $24^{\circ}$ Dornic ramené à $18^{\circ}$ ) l'extrait sec est passé de 130,0 à 126,9 , soit une perte de 3 grammes par litre (toutes précautions étant prises pour éviter l'influence perturbatrice de la mousse).

Mais en fait, pratiquement, on retire de l'appareil après l'opération $3 \mathrm{~kg}$. 600 de caillebotte brute non pressée pour 200 litres de lait traités. Dans le cas d'un traitement plus intense (lait à $40^{\circ} \mathrm{D}$. ramené à $24^{\circ}$ ) le dépôt de caillebotte brute formé dans l'appareil et entre les plaques a été trouvé égal à $18 \mathrm{~kg}$. 400 pour 200 litres de lait. Il s'agit bien entendu de caillebotte imprégnée de lait.

Ce sont là des pertes de matière importantes qu'il nous paraît bien difficile de réduire.

\section{$3^{\circ}$ Matière grasse.}

La perte de matière grasse constatée au cours des opérations est très variable suivant l'intensité du traitement. Cette perte est due à l'entraînement mécanique des globules gras par la caséine au cours de la précipitation.

A vrai dire cette perte est assez minime si le traitement est ménagé. Exemple : on passe de 40 gr. 5 à 40 grammes de matière grasse par litre pour une désacidification de $24^{\circ} \mathrm{D}$. à $18^{\circ}$. Mais elle est très notable si la désacidification est importante comme ce serait le cas en fromagerie. Ainsi au cours d'une désacidification de $40^{\circ} \mathrm{D}$. ̀̀ $24^{\circ}$ la matière grasse du lait passe de 40 grammes à 34 grammes par litre.

\section{$4^{\circ}$ Extrait dégraissé.}

Il subit une chute influencée surtout par la précipitation partielle de la caséine. C'est ainsi que dans le cas le plus favorable d'un traitement très léger (de 24 à $18^{\circ} \mathrm{D}$. par exemple) l'extrait dégraissé passe de 89,5 à 86,9 , soit une perte de 2 gr. 6 par litre. Cette perte est la même que celle qui résulterait d'un mouillage de 2,5 à $3 \%$. Il va de soi que pour des désacidifications plus importantes cet inconvénient s'aggrave dans des proportions inquiétantes.

\section{Densité.}

Elle est moins influencée que l'extrait parce que la caséine qui 
précipite (diminution de densité) entraîne avec elle de la matière grasse (augmentation de densité). Ces deux influences tendent à se compenser. Néanmoins la perte de caséine étant toujours plus importante que celle de la matière grasse, le résultat final se traduit par une légère diminution de la densité.

Exemple : pour une désacidification faible $\left(24\right.$ à $18^{\circ} \mathrm{D}$. par exemple) la densité est à peine touchée ; on passe de 1031,2 à 1031,1 . Alors que dans le cas d'une désacidification importante (de 40 à $24^{\circ}$ D.) la densité passe par exemple de 1031,3 à 1031,0 .

\section{$6^{\circ}$ Composition bactériologique.}

En dépit de ce qui est généralement annoncé par les promoteurs de ce procédé, le nombre de germes vivants du lait ne semble modifié par le traitement. En particulier, le nombre de colibacilles n'a pas diminué au cours des quelques expériences que nous avons exécutées.

\section{Conservabilité.}

Nous avons suivi l'acidification spontanée du lait cru désacidifié électriquement et du lait cru témoin. Nous avons toujours constaté une réacidification très rapide du lait cru désacidifié (aussi rapide et souvent plus rapide que celle du lait cru non traité).

Bien entendu, si ces laits sont soumis à la pasteurisation, leur conservabilité s'améliore dans des proportions très considérables ; mais il en est de même pour les laits non traités (dans le cas où leur acidité est assez faible pour permettre la pasteurisation). Et si l'on se contente de noter avec certains auteurs que le lait désacidifié et pasteurisé se conserve mieux que le lait cru non désacidifié, on n'a pas le droit d'en reporter le mérite exclusif sur cette désacidification électrique. C'est évidemment la pasteurisation qui est la cause de l'amélioration de conservation constatée ; et le lait cru non traité électriquement est justiciable du même progrès par la pasteurisation pure et simple (à égalité d'acidité au début de l'observation, cela va de soi).

\section{$8^{\circ}$ Examen organoleptique.}

Après le traitement électrique le lait recueilli dans le cas d'un traitement ménagé, c'est-à dire avec un passage du lait assez rapide, paraît normal. A froid il n'a pas d'odeur particulière et sa saveur n'est pas sensiblement modifiée (sauf en ce qui concerne la diminution d'acidité).

Mais si le traitement a été plus intense, si on est descendu nettement au-dessous de l'acidité naturelle du lait, celui-ci est profondément altéré : le goût est désagréable ; chauffé il répand une odeur de poisson qui traduit une profonde décomposition des protéines. 
Une aération modérée n'élimine pas complètement cette odeur insolite.

\section{IDEE D'UNE THÉORIE DE LA DÉSACIDIFICATION ELECTROLYTIQUE DU LAIT}

On connaît un certain nombre de faits expérimentaux qui peuvent servir de base à une théorie au moins provisoire du phénomène étudié.

Ces faits sont les suivants :

$1^{0}$ Une solution d'acide lactique soumise à l'électrolyse ne voit pas son acidité potentielle diminuer. Au contraire cette acidité augmente peu à peu. Tout se passe comme si l'acide lactique se scindait en acide formique et aldéhyde acétique susceptible d'être oxydé à l'anode en acide acétique.

On aurait :

$\mathrm{CH}^{3}$. $\mathrm{CHOH} . \mathrm{COOH} \rightarrow \mathrm{H} \cdot \mathrm{COOH}+\mathrm{CH}^{3}$. $\mathrm{CHO}$ qui $+\mathrm{O} \rightarrow \mathrm{CH}^{3}$. $\mathrm{COOH}$

Ce fait d'expérience (augmentation de l'acidité pendant l'électrolyse) s'oppose donc à l'idée que la désacidification pourrait être due à la destruction de l'acide lactique.

D'ailleurs nous avons vu que, dans les conditions de la désacidification électrique du lait, les radicaux lactiques étaient conservés.

Enfin, comment expliquerait-on la désacidification de laits frais exempts d'acide lactique et, a fortiori, la neutralisation et l'alcalinisation du lait sous l'influence du courant?

Done, ce n'est pas une destruction électrolytique de l'acide lactique qui cause la désacidification du lait.

$2^{\circ}$ L'électrolyse de la solution d'un sel métallique (par exemple phosphate neutre) avec des électrodes d'aluminium provoque rapidement une alcalinisation de la solution.

Il se forme à la cathode une base libre (soude, potasse, chaux). A l'anode, l'anion (l'acide phosphorique libéré par exemple) réagit sur le métal de l'électrode et son acidité se trouve bloquée. Nous avons même constaté dans le cas actuel la formation anodique d'un précipité de phosphate d'aluminium.

La réaction pourrait s'exprimer globalement de la manière suivante :

$$
\begin{aligned}
\mathrm{Al}^{2}+\left(\mathrm{PO}^{4}\right)^{2}\left(\mathrm{M}_{+++}\right)^{3}+6 \mathrm{CH}^{3} \cdot \mathrm{CHOH} . \mathrm{COOH}= \\
=\left(\mathrm{PO}^{4}\right)^{2} \cdot \mathrm{Al}^{2}+3\left(\mathrm{CH}^{3} \cdot \mathrm{CHOH} . \mathrm{COO}\right)^{2} \cdot \mathrm{M}_{++} \cdot
\end{aligned}
$$

Au total donc, alcalinisation.

Le même raisonnement est d'ailleurs identiquement valable avec d'autres sels.

L'un des arguments les meilleurs en faveur de l'exactitude de cette hypothèse dans le cas de la désacidification du lait, est la 
présence abondante d'aluminium dans le dépôt de caillebotte qui se forme aux anodes.

$3^{\circ}$ L'électrolyse du lait est toujours accompagnée d'une précipitation de caséine.

Grâce à ces trois faits, on peut s'expliquer la désacidification éleetrique du lait de la manière suivante :

a) D'abord, nous l'avons dit, une partie de la désacidification s'explique purement et simplement par la précipitation à l'anode de la caséine, colloïde électro-négatif.

b) Puis et surtout, par la libération de bases alcalino-terreuses principalement aux dépens des phosphates du lait (et même des phosphocaséinates) dont l'anion se fixe sur le métal des électrodes. Les bases ainsi libérées neutralisent l'acide lactique libre s'il en existe, puis une partie de la caséine, et peuvent même si elles sont assez abondantes aller jusqu'à rendre le lait complètement alcalin.

Un calcul simple montre que les phosphates théoriques contenus dans un lait normal représentent, par leurs bases, une réserve d'alcalinité en puissance capable de saturer jusqu'à environ $50^{\circ} \mathrm{D}$. Si done l'électrolyse est poussée assez loin dans un lait titrant au départ moins de $50^{\circ} \mathrm{D}$. on peut arriver à réaliser l'alcalinité dans ce lait. C'est ce que l'expérience nous a permis de confirmer (nous avons atteint et dépassé le point permettant au lait de faire virer la phtaléine au rouge).

Selon nous, le mécanisme de la désacidification électrique du lait serait donc en grande partie basé sur l'électrolyse des phosphates, le cathion de ceux-ci jouant le rôle d'agent neutralisant, et l'anion s'insolubilisant par combinaison avec le métal des électrodes.

Si on poursuit l'électrolyse après que toutes les bases libérables des phosphates ont saturé l'acide lactique, autrement dit si on effectue l'électrolyse en milieu neutre ou déjà neutralisé, on peut penser que de nouvelles réactions vont apparaître, notamment des coupures dans la molécule de caséine dues par exemple à l'action de nouvelles bases libérées et commençant à hydrolyser la caséine.

Ces coupures avec apparition de polypeptides, voire d'acides aminés, pourraient être rendues responsables de l'odeur spéciale du lait soumis à une électrolyse profonde.

\section{AVANTAGES ET INCONVÉNIENTS DE LA DÉSACIDIFICATION ÉLECTRIQUE}

De cette courte étude d'ailleurs très incomplète, il résulte :

10 Que le procédé de désacidification électrique permet effectivement de réduire l'acidité d'un lait quelconque dans des proportions fort variables. 
$2^{\circ} \mathrm{Qu}$ 'il est possible de faire descendre l'acidité d'un lait quelconque en-dessous de l'acidité naturelle d'un lait frais et qu'on peut même préparer du lait nettement alcalin.

3. Que cette désacidification, à elle seule, ne confère pas aux laits auxquels on l'applique des propriétés spéciales vis-à-vis de la réacidification ultérieure.

$4^{\circ}$ Que cette opération ne modifie pas d'une façon sensible la flore bactérienne du lait.

5o Que la désacidification s'accompagne d'une précipitation de caséine en quantité non négligeable. Cette perte s'accompagne d'une disparition moins importante de matière grasse. Il en résulte, même dans les cas les plus favorables, une diminution de l'extrait sec dégraissé assez notable pour conférer aux laits certains aspects chimiques du lait mouillé.

$6^{\circ}$ Que ce traitement communique au lait dans certains cas une odeur et une saveur très désagréables exaltées par la pasteurisation et non complètement éliminées par l'aération.

Nous n'avons pas expérimenté l'utilisation éventuelle de laits alcalinisés électriquement, à la réduction de l'acidité des crèmes ou au lavage des beurres acides.

Les essais relatés ci-dessus nous permettent simplement cette conclusion que le procédé de désacidification électrique ne nous paraît pas applicable au traitement du lait destiné à la vente en nature et cela pour des raisons d'ordre légal, économique et commercial.

\section{BIBLIOGRAPHIE}

[1] Otito Gratz (traduction de M. Ǵenin). Le Lait, février 1934, p. 145.

[2] W. WINKLER. Le Lait, mai 1935, p. 505

\section{LES AMÉLIORANTS DES BEURRES A BASE DE DIACÉTYLE SONT-ILS DES PRODUITS NATURELS ? (1)}

par

M. LEMOIGNE

Professeur à l'Institut National

Agronomique. et P. MONGUILLON

Maître de Conférences à l'Institut

National Agronomique.

Après tant d'autres, un nouvel améliorant du beurre a été proposé depuis quelques années sous divers noms. Il se présente sous forme de lait caillé contenant, d'après les quelques analyses que nous avons pu faire, au moins 5 à 7 grammes par litre de diacétyle ( $\left.\mathrm{CH}^{3}-\mathrm{CO}-\mathrm{CO}-\mathrm{CH}^{3}\right)$.

(1) Annales des Falsifications et des Fraudes, n०317, mai 1935, p. 278. 